FACTA UNIVERSITATIS (NIŠ)

Ser. Math. Inform. Vol. 36, No 3 (2021), 585 - 593

https://doi.org/10.22190/FUMI201225043I

Original Scientific Paper

\title{
ON SOME EQUIVALENCE RELATION ON NON-ABELIAN CA-GROUPS
}

\author{
Mohammad A. Iranmanesh and Mohammad Hossein Zareian
}

Department of Mathematical Science, Yazd University

P. O. Box 89158-741, Yazd, Iran

\begin{abstract}
A non-abelian group $G$ is called a CA-group (CC-group) if $C_{G}(x)$ is abelian (cyclic) for all $x \in G \backslash Z(G)$. We say $x \sim y$ if and only if $C_{G}(x)=C_{G}(y)$. We denote the equivalence class including $x$ by $[x]_{\sim}$. In this paper, we prove that if $G$ is a CA-group

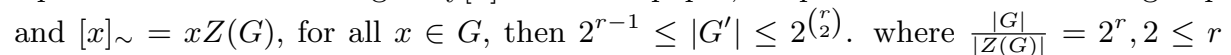
and characterize all groups whose $[x]_{\sim}=x Z(G)$ for all $x \in G$ and $|G| \leq 100$. Also, we will show that if $G$ is a CC-group and $[x]_{\sim}=x Z(G)$, for all $x \in G$, then $G \cong C_{m} \times Q_{8}$ where $C_{m}$ is a cyclic group of odd order $m$ and if $G$ is a CC-group and $[x]_{\sim}=x^{G}$, for all $x \in G \backslash Z(G)$, then $G \cong Q_{8}$.
\end{abstract}

Keywords: CA-group, CC-group, centralizer of a group, derived subgroup.

\section{Introduction}

Throughout this paper all groups are assumed to be finite. We denote by $Z(G)$, $C_{G}(x), \operatorname{Cent}(G),|\operatorname{Cent}(G)|, x^{G}, G^{\prime}$ and $k(G)$ the center of the group $G$, the centralizer of $x \in G$, the set of centralizers of the group $G$, the number of centralizers of the group $G$, the conjugacy class of $x \in G$, the derived subgroup of the group $G$, the number of conjugacy classes of the group $G$, respectively. The authors in [8], denoted by $[m, n]$ the GAP ID of a group which is a label that uniquely identifies a group in GAP. The first number in $[m, n]$ is the order of the group, and the second number simply enumerates different groups of the same order. We will use usual notation, for example $C_{n}, D_{2 n}$ and $Q_{2^{n}}$ denote the cyclic group of order $n$, the

Received December 25, 2020. accepted January 17, 2021.

Communicated by Alireza Ashrafi, Hassan Daghigh

Corresponding Author: Mohammad A. Iranmanesh, Department of Mathematical Science, Yazd

University, P. O. Box 89158-741, Yazd, Iran | E-mail: iranmanesh@yazd.ac.ir

2010 Mathematics Subject Classification. Primary xxxxx; Secondary xxxxx, xxxxx

(C) 2021 by University of Niš, Serbia | Creative Commons License: CC BY-NC-ND 
dihedral group of order $2 n$ and the generalized quaternion group of order $2^{n}$ respectively. The non-commuting graph $\Gamma(G)$ with respect to $G$ is a graph with vertex set $G \backslash Z(G)$ and two distinct vertices $x$ and $y$, are adjacent whenever $[x, y] \neq 1$. A non-abelian group $G$ is called a CA-group (CC-group) if $C_{G}(x)$ is abelian (cyclic) for all $x \in G \backslash Z(G)$. We say $x \sim y$ if and only if $C_{G}(x)=C_{G}(y)$, and $x \sim_{1} y$ if and only if $x Z(G)=y Z(G)$. We denote the equivalence class including $x$ under $\sim$ by $[x]_{\sim}$. The number of equivalence classes of $\sim$ and $\sim_{1}$ on the group $G$ are equal with $|\operatorname{Cent}(G)|$ and $\frac{|G|}{|Z(G)|}$ respectively. The influence of $|\operatorname{Cent}(G)|$ on the group $G$ has been investigated in $[3,2,4]$. In [5], CA-groups whose $[x]_{\sim}=x Z(G)$ for all $x \in G$ has been investigated. In this paper we have investigated the equivalency of above relations. We will use the following lemmas to prove the main theorems.

Lemma 1.1. [1, Lemma 3.6] Let $G$ be a non-abelian group. Then the following are equivalent:

1) $G$ is a CA-group.

2) If $[x, y]=1$ then $C_{G}(x)=C_{G}(y)$, where $x, y \in G \backslash Z(G)$.

3) If $[x, y]=[x, z]=1$ then $[y, z]=1$, where $x \in G \backslash Z(G)$.

4) If $A, B \leq G, Z(G) \supsetneqq C_{G}(A) \leqslant C_{G}(B) \supsetneqq G$, then $C_{G}(A)=C_{G}(B)$.

Lemma 1.2. [1, Proposition 2.6] Let $G$ be a finite non-abelian group and $\Gamma(G)$ be a regular graph. Then $G$ is nilpotent of class at most 3 and $G=A \times P$, where $A$ is an abelian group and $P$ is a p-group ( $p$ is a prime) and furthermore $\Gamma(P)$ is a regular graph.

Lemma 1.3. [5, Lemma 11] Let $G$ be a non-abelian group. Then $x Z(G) \subseteq[x]_{\sim}$, for all $x \in G$. Also the equality happens if and only if $|\operatorname{Cent}(G)|=\frac{|G|}{|Z(G)|}$.

Lemma 1.4. [5, Lemma 12] Let $G$ be a finite non-abelian group. Then the following are equivalent:

1) If $[x, y]=1$, then $x Z(G)=y Z(G)$, where $x, y \in G \backslash Z(G)$.

2) $G$ is a CA-group and $[x]_{\sim}=x Z(G)$, for all $x \in G$.

3) $[x, y]=1$ and $[x, w]=1$ imply that $y Z(G)=w Z(G)$, where $x, y, w \in G \backslash Z(G)$.

Lemma 1.5. [5, Theorem 3] Let $G$ be a non-abelian group. The following are equivalent:

1) $G$ is a CA-group and $|\operatorname{Cent}(G)|=\frac{|G|}{|Z(G)|}$.

2) $G=A \times P$, where $A$ is an abelian group, $P$ is a 2-group, $P$ is a CA-group and $|\operatorname{Cent}(P)|=\frac{|P|}{|Z(P)|}$. 
3) $G=A \times P$, where $A$ is an abelian group and $C_{P}(x)=Z(P) \cup x Z(P)$, for all $x \in P \backslash Z(P)$.

Lemma 1.6. [5, Lemma 13] Let $G$ be a non-abelian group. Let $[x]_{\sim}$ and $[y]_{\sim}$ be two different classes of $\sim$. If $\left[x_{0}, y_{0}\right] \neq 1$ for some $x_{0} \in[x]_{\sim}$ and $y_{0} \in[y]_{\sim}$, then $[u, v] \neq 1$ for all $u \in[x]_{\sim}$ and $v \in[y]_{\sim}$.

Lemma 1.7. [5, Lemma 20] Let $G_{1}$ and $G_{2}$ be two groups. Let $\left[g_{1}\right]_{\sim}=g_{1} Z\left(G_{1}\right)$, for all $g_{1} \in G_{1}$ and $\left[g_{2}\right]_{\sim}=g_{2} Z\left(G_{2}\right)$, for all $g_{2} \in G_{2}$. Then $[X]_{\sim}=X Z\left(G_{1} \times G_{2}\right)$, for all $X \in G_{1} \times G_{2}$.

Lemma 1.8. [6, Theorem 2.1] Let $G$ be a non-abelian group and $|\operatorname{Cent}(G)|=$ $\frac{|G|}{|Z(G)|}$. Then $\frac{G}{Z(G)}$ is an elementary abelian 2-group.

Lemma 1.9. [7, Corollary 2.3] Let $G$ be a non-abelian nilpotent group. Then $G$ is a CC-group if and only if $G \cong C_{m} \times Q_{2^{n}}$, where $m$ and $n$ are positive integers and $m$ is odd.

In Section 2 we will provide some results about the equivalency of relations.

\section{Proof of the main theorems}

In this section we prove the main theorems. For doing this we first prove some lemmas.

Lemma 2.1. Let $G$ be a CA-group. Then $C_{G}(x)=Z(G) \cup[x]_{\sim}$, for all $x \in$ $G \backslash Z(G)$.

Proof. Since $Z(G) \subseteq C_{G}(x)$ and $[x]_{\sim} \subseteq C_{G}(x)$ we have $Z(G) \cup[x]_{\sim} \subseteq C_{G}(x)$. Suppose $g \in C_{G}(x) \backslash Z(G)$. Then $[g, x]=1$. By Lemma 1.1, $C_{G}(x)=C_{G}(g)$ which implies that $[x]_{\sim}=[g]_{\sim}$. Hence $g \in[x]_{\sim}$ and we have $C_{G}(x) \subseteq Z(G) \cup[x]_{\sim}$. Therefore $C_{G}(x)=Z(G) \cup[x]_{\sim}$, for all $x \in G \backslash Z(G)$.

Lemma 2.2. Let $G$ be a non-abelian group. Then $G$ is a CA-group and $[x]_{\sim}=$ $x Z(G)$, for all $x \in G$ if and only if $|G|=\frac{2|Z(G)|^{2}}{(3|Z(G)|-k(G))}$.

Proof. Let $G$ be a CA-group and $[x]_{\sim}=[x]_{\sim_{1}}$, for all $x \in G$. Let $x Z(G) \neq$ $y Z(G)$ for some $x, y \in G \backslash Z(G)$. Since $X Y \neq Y X$ for all $X \in x Z(G)$ and $Y \in$ $y Z(G)$, therefore there exists an edge between $X$ and $Y$. Hence there are $|Z(G)|^{2}$ edges between elements of $x Z(G)$ and $y Z(G)$. Also there are $\frac{|G|}{|Z(G)|}-1$ different classes of $x Z(G)$ for $x \in G \backslash Z(G)$. Thus $|E(\Gamma(G))|=\left(\frac{|G|}{\left|\frac{|G|}{2}\right|-1}\right)|Z(G)|^{2}$. Note that by [1, Lemma 3.27], $|E(\Gamma(G))|=\frac{|G|^{2}-k(G)|G|}{2}$. Hence $|G|=\frac{2|Z(G)|^{2}}{3|Z(G)|-k(G)}$.

Conversely, suppose $|G|=\frac{2|Z(G)|^{2}}{3|Z(G)|-k(G)}$. So $|G|=|Z(G)|+(k(G)-|Z(G)|) \frac{|G|}{2|Z(G)|}$. Since for all $x \in G \backslash Z(G),\left|x^{G}\right| \leq \frac{|G|}{2 \mid Z(G)}$ we have $\left|x^{G}\right|=\frac{|G|}{2|Z(G)|}$, for all $x \in G \backslash Z(G)$. 
So $\left|C_{G}(x)\right|=2|Z(G)|$, for all $x \in G \backslash Z(G)$. Now by [5, Lemma 15] $G$ is a CA-group and $[x]_{\sim}=[x]_{\sim_{1}}$.

Example 2.1. Let $G$ be a CA-group and $[x]_{\sim}=x Z(G)$, for all $x \in G$ and $|G| \leq 100$. Then $G$ is one of the group with GAP ID in Table 2.1.

Table 2.1: The GAP ID of group $G$ where $|G|=\frac{2|Z(G)|^{2}}{3|Z(G)|-k(G)}$ and $|G| \leq 100$.

\begin{tabular}{|c|c|c|c|c|c|c|c|}
\hline$[8,3]$ & {$[8,4]$} & & & & & & \\
\hline$[16,3]$ & {$[16,4]$} & {$[16,6]$} & {$[16,11]$} & {$[16,12]$} & {$[16,13]$} & & \\
\hline$[24,10]$ & {$[24,11]$} & & & & & & \\
\hline 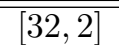 & $\overline{[32,4]}$ & $\overline{[32,5]}$ & $\overline{[}[32,12]$ & $\overline{[32,17]}$ & {$[32,22]$} & {$[32,23]$} & 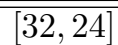 \\
\hline$[32,25]$ & {$[32,26]$} & {$[32,37]$} & {$[32,38]$} & {$[32,46]$} & {$[32,47]$} & {$[32,48]$} & \\
\hline$[40,11]$ & {$[40,12]$} & & & & & & \\
\hline$[48,21]$ & {$[48,22]$} & $48,24]$ & {$[48,45]$} & {$[48,46]$} & {$[48,47]$} & & \\
\hline$[56,9]$ & {$[56,10]$} & & & & & & \\
\hline 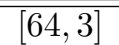 & 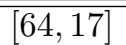 & $\begin{array}{l}64,27] \\
\end{array}$ & 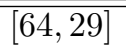 & $\overline{[64,44]}$ & 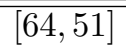 & 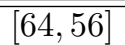 & 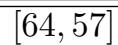 \\
\hline$[64,58]$ & {$[64,59]$} & {$[64,73]$} & {$[64,74]$} & {$[64,75]$} & {$[64,76]$} & {$[64,77]$} & {$[64,78]$} \\
\hline$[64,79]$ & {$[64,80]$} & {$[64,81]$} & {$[64,82]$} & {$[64,84]$} & {$[64,85]$} & {$[64,86]$} & {$[64,87]$} \\
\hline$[64,103]$ & {$[64,112]$} & {$[64,115]$} & {$[64,126]$} & {$[64,184]$} & {$[64,185]$} & {$[64,193]$} & {$[64,194$} \\
\hline$[64,195]$ & {$[64,196]$} & {$[64,197]$} & {$[64,198]$} & {$[64,247]$} & {$[64,248]$} & {$[64,261]$} & {$[64,262$} \\
\hline \multicolumn{8}{|l|}{$[64,263]$} \\
\hline$[72,10]$ & {$[72,11]$} & 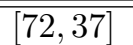 & {$[72,38]$} & & & & \\
\hline$[80,21]$ & {$[80,22]$} & {$[80,24]$} & {$[80,46]$} & {$[80,47]$} & {$[80,48]$} & & \\
\hline$[88,9]$ & {$[88,10]$} & & & & & & \\
\hline$[96,45]$ & {$[96,47]$} & {$[96,48]$} & {$[96,52]$} & {$[96,54]$} & {$[96,55]$} & {$[96,60]$} & {$[96,162$} \\
\hline$[96,163]$ & {$[96,165]$} & {$[96,166]$} & {$[96,167]$} & {$[96,221]$} & {$[96,222]$} & {$[96,223]$} & \\
\hline
\end{tabular}

Theorem 2.1. Let $G$ be a CA-group and $[x]_{\sim}=x Z(G)$, for all $x \in G$. Then $2^{r-1} \leq\left|G^{\prime}\right| \leq 2^{\left(\begin{array}{c}r \\ 2\end{array}\right)}$, where $\frac{|G|}{|Z(G)|}=2^{r}, 2 \leq r$.

Proof. Let $G$ be a CA-group and $[x]_{\sim}=x Z(G)$, for all $x \in G$. First we show

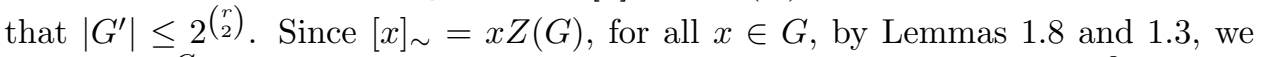
find that $\frac{G}{Z(G)}$ is an elementary abelian 2-group. Therefore $G^{\prime} \leq Z(G), g^{2} \in Z(G)$, for all $g \in G$ and $G^{\prime}$ is an elementary abelian 2-group. Since $G$ is a non-abelian group, there exist $x, y \in G$ such that $[x, y]=z \neq 1$ and $[x, x y] \neq 1$ and $[y, x y] \neq 1$. By Lemma 1.4, $x Z(G) \neq y Z(G), x Z(G) \neq x y Z(G)$ and $y Z(G) \neq x y Z(G)$. Let $H_{1}=Z(G) \cup x Z(G) \cup y Z(G) \cup x y Z(G)$. Since $\frac{G}{Z(G)}$ is an elementary abelian 2group, $H_{1} \leq G$. By Lemma 1.6, none of the elements of $x Z(G)$ are commute with elements of $y Z(G)$ and $x y Z(G)$. Also none of the elements of $y Z(G)$ are commute 
with elements of $x y Z(G)$. Therefore $Z\left(H_{1}\right)=Z(G)$. Since $G^{\prime} \leq Z(G)$ and $t^{2}=1$, for all $t \in G^{\prime}$, we have the following:

$$
[x, y]^{-1}=[y, x]=[x, y]=[x, x y]=[y, y x]=z,[e u, f w]=[e, f],
$$

for all $e, f \in\{x, y, x y\}$ and for all $u, w \in Z(G)$. Hence

$$
\begin{gathered}
H_{1}^{\prime}=\left\langle\left[g_{1}, h_{1}\right] \mid g_{1}, h_{1} \in H_{1}\right\rangle=\langle[e u, f w] \mid e, f \in\{x, y, x y\}, u, w \in Z(G)\rangle \\
=\langle[e, f] \mid e, f \in\{x, y, x y\}\rangle=\langle[x, y]\rangle=\langle z\rangle=\{1, z\} .
\end{gathered}
$$

Thus $\left|H_{1}^{\prime}\right|=2 \leq 2^{\left(\begin{array}{l}2 \\ 2\end{array}\right)}$ and $\frac{\left|H_{1}\right|}{\left|Z\left(H_{1}\right)\right|}=\frac{4|Z(G)|}{|Z(G)|}=2^{2}$. If $G=H_{1}$ then proof is complete, so assume that $G \neq H_{1}$. Hence there exists $a \in G \backslash H_{1}$. Let $H_{2}=H_{1}\langle a\rangle$. Since $a^{2} \in Z(G)$ we have

$$
\begin{aligned}
H_{2}=\quad H_{1}\langle a\rangle & =H_{1} \cup a H_{1}=Z(G) \cup x Z(G) \cup y Z(G) \cup x y Z(G) \\
& \cup a Z(G) \cup a x Z(G) \cup a y Z(G) \cup a x y Z(G)
\end{aligned}
$$

and since $\frac{G}{Z(G)}$ is an elementary abelian 2-group, $H_{2} \leq G$. By Lemma $1.6 Z\left(H_{2}\right)=$ $Z(G)$. Let $[a, x]=t_{1},[a, y]=t_{2}$. Therefore $1 \neq[a, x y]=[a, x][a, y]=t_{1} t_{2}$. In above we had $[x, y]=[x, x y]=[y, x y]=z$. On the other hand $\left[e_{1} u, f_{1} w\right]=\left[e_{1}, f_{1}\right]$, for all $u, w \in Z(G)$ and for all $e_{1}, f_{1} \in\{x, y, x y, a, a x, a y, a x y\}$. Also $\left[g_{2}, h_{2} k_{2}\right]=$ $\left[g_{2}, h_{2}\right]\left[g_{2}, k_{2}\right]$, for all $g_{2}, h_{2}, k_{2} \in H_{2}$. Hence

$$
\begin{gathered}
H_{2}^{\prime}=\left\langle\left[g_{2}, h_{2}\right] \mid g_{2}, h_{2} \in H_{2}\right\rangle=\left\langle\left[e_{1} u, f_{1} w\right] \mid e_{1}, f_{1} \in\{x, y, x y, a, a x, a y, a x y\}\right\rangle \\
=\langle[x, y],[a, x],[a, y]\rangle=\left\langle z, t_{1}, t_{2}\right\rangle .
\end{gathered}
$$

Therefore $\left|H_{2}^{\prime}\right| \leq 2^{\left(\begin{array}{l}3 \\ 2\end{array}\right)}$ and $\frac{\left|H_{2}\right|}{\left|Z\left(H_{2}\right)\right|}=\frac{8|Z(G)|}{|Z(G)|}=2^{3}$. If $G=H_{2}$, then the proof is complete. Let $G \neq H_{2}$. Therefore there exists $b \in G \backslash H_{2}$. Let $H_{3}=H_{2}\langle b\rangle$. Let $[b, x]=l_{1},[b, y]=l_{2},[b, a]=l_{3}$. By a Similar calculation we have, $Z\left(H_{3}\right)=Z(G)$ and $H_{3}^{\prime}=\left\langle z, t_{1}, t_{2}, l_{1}, l_{2}, l_{3}\right\rangle$. Hence $\left|H_{3}^{\prime}\right| \leq 2^{6}=2^{\left(\begin{array}{c}4 \\ 2\end{array}\right)}$ and $\frac{\left|H_{3}\right|}{\left|Z\left(H_{3}\right)\right|}=\frac{16|Z(G)|}{|Z(G)|}=2^{4}$. By continuing this process, we have the following subgroups: $Z(G) \leq H_{1} \leq H_{2} \leq$

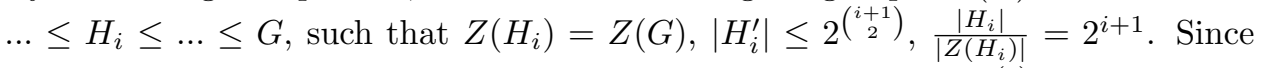
$G$ is finite, there exists $2 \leq r$, such that $G=H_{r-1},\left|G^{\prime}\right| \leq 2^{\left(\begin{array}{c}r \\ 2\end{array}\right)}$ and $\frac{|G|}{|Z(G)|}=$ $\frac{\left|H_{r-1}\right|}{\left|Z\left(H_{r-1}\right)\right|}=2^{r}$. Since $[w]_{\sim}=w Z(G)$, for all $w \in G \backslash Z(G)$, so by Lemma 2.1, $\left|w^{G}\right|=\frac{|G|}{\left|C_{G}(w)\right|}=\frac{|G|}{2|Z(G)|}$, for all $w \in G \backslash Z(G)$. Consequently, as $w^{G} \subseteq w G^{\prime}$, we have $\frac{|G|}{2|Z(G)|}=2^{r-1} \leq\left|G^{\prime}\right|$.

Theorem 2.2. Let $G$ be a non-abelian CC-group and $[x]_{\sim}=x Z(G)$, for all $x \in G$. Then $G \cong C_{m} \times Q_{8}$ where $C_{m}$ is a cyclic group of odd order $m$.

Proof. Let $G$ be a CC-group and $[x]_{\sim}=x Z(G)$, for all $x \in G$. Therefore $G$ is a CA-group. By lemma 1.3, $|\operatorname{Cent}(G)|=\frac{|G|}{|Z(G)|}$ and by lemma $1.5, G \cong A \times P$ where $A$ is an abelian group and $P$ is a 2-group. Hence $G$ is a nilpotent group. By lemma 
$1.9, G \cong C_{m} \times Q_{2^{n}}$ where $C_{m}$ is a cyclic group of order odd $m$. Since $[x]_{\sim}=x Z(G)$ for all $x \in G$, we have by lemma 1.3 , that $|\operatorname{Cent}(G)|=\frac{|G|}{|Z(G)|}$ and by Lemma $1.8, \frac{G}{Z(G)}$ is an elementary abelian 2-group which implies that $G^{\prime} \leq Z(G)$. Hence $\left(C_{m} \times Q_{2^{n}}\right)^{\prime} \subseteq Z\left(C_{m} \times Q_{2^{n}}\right)$ and $1 \times Q_{2^{n}}^{\prime} \subseteq C_{m} \times Z\left(Q_{2^{n}}\right) \cong C_{m} \times C_{2}$. Therefore $Q_{2^{n}}^{\prime} \cong C_{2}$ and $\left|Q_{2^{n}}^{\prime}\right|=2$. Since $\left|Q_{2^{n}}^{\prime}\right|=2^{n-2}$, we have $n=3$ and $G \cong C_{m} \times Q_{8}$.

Conversely $Q_{8}$ is a CC-group and $[x]_{\sim}=x Z(G)$, for all $x \in G$. Therefore $C_{m} \times Q_{8}$ is also a CC-group and by Lemma 1.7, $[x]_{\sim}=x Z(G)$ for all $x \in G \cong$ $C_{m} \times Q_{8}$.

Proposition 2.1. Let $G$ be a non-abelian group and $G^{\prime} \leq Z(G)$. Then if $[x]_{\sim}=$ $x^{G}$, for all $x \in G \backslash Z(G)$ then $[x]_{\sim}=x^{G}=x Z(G)$, for all $x \in G \backslash Z(G)$ and $G^{\prime}=Z(G)$.

Proof. Let $[x]_{\sim}=x^{G}$, for all $x \in G \backslash Z(G)$. Since $G^{\prime} \leq Z(G)$, so $x G^{\prime} \leq x Z(G)$. By Lemma $1.3, x Z(G) \subseteq[x]_{\sim}$, for all $x \in G$. Hence $x Z(G) \subseteq[x]_{\sim}=x^{G} \subseteq x G^{\prime} \subseteq$ $x Z(G)$, for all $x \in G \backslash Z(G)$. This implies that $[x]_{\sim}=x^{G}=x G^{\prime}=x Z(G)$, for all $x \in G \backslash Z(G)$. Since $\left|x G^{\prime}\right|=|x Z(G)|$ we have $G^{\prime}=Z(G)$ and the proof is complete.

Example 2.2. Let $G$ be an extra especial group of order 32 . Then $[x]_{\sim}=x^{G}=x Z(G)$, for all $x \in G \backslash Z(G)$.

Theorem 2.3. Let $G$ be a CA-group and $[x]_{\sim}=x^{G}$, for all $x \in G \backslash Z(G)$. Then $G$ is a 2-group, $\frac{G}{Z(G)}$ is an elementary abelian 2-group, $[x]_{\sim}=x^{G}=x Z(G)$, for all $x \in G \backslash Z(G)$ and $G^{\prime}=Z(G)$.

Proof. Since $G$ is a CA-group, by Lemma 2.1, $C_{G}(x)=[x]_{\sim} \cup Z(G)$, for all $x \in G \backslash Z(G)$. Therefore $\left|x^{G}\right|=\frac{|G|}{\left|C_{G}(x)\right|}=\frac{|G|}{|Z(G)|+|[x] \sim|}=\frac{|G|}{|Z(G)|+\left|x^{G}\right|}$ which implies that $\left|x^{G}\right|^{2}+|Z(G)|\left|x^{G}\right|-|G|=0$. So $\left|x^{G}\right|$ is a constant and $\Gamma(G)$ is a regular graph. By Lemma 1.2, $G=A \times P$ where $A$ is an abelian group and $P$ is a $p$-group ( $p$ is a prime) and by Lemma $1.3, x Z(G) \subseteq[x]_{\sim}$, for all $x \in G \backslash Z(G)$. Therefore $x Z(G) \subseteq[x]_{\sim}=x^{G} \subseteq x G^{\prime}$ which implies that $x Z(G) \subseteq x G^{\prime}$. Thus $Z(G) \leq G^{\prime}$ and $Z(G)=A \times Z(P) \leq G^{\prime}=1 \times P^{\prime}$. Hence $A \cong 1$ and $Z(P) \leq P^{\prime}$. So $G$ is a $p$-group and $G \cong P$ and there exist positive integers $m, n, t$ so that $|P|=$ $p^{n},|Z(P)|=p^{t},\left|x^{P}\right|=p^{m}$ and $p^{m}=\frac{p^{n}}{\left(p^{t}+p^{m}\right)}$. This implies that $p^{2 m}+p^{t+m}=p^{n}$ and $p^{m-t}+1=p^{n-m-t}$. Since $p$ is a prime, by discussing the different states of the prime numbers, we obtain $p=2$ and $m=t$. Since $x Z(P) \subseteq[x]_{\sim}=x^{P}$ and $\left|x^{P}\right|=|Z(P)|$, so $[x]_{\sim}=x^{P}=x Z(P)$, for all $x \in P \backslash Z(P)$. By Lemma 1.3, $|\operatorname{Cent}(P)|=\frac{|P|}{|Z(P)|}$. This implies by Lemma 1.8, that $\frac{P}{Z(P)}$ is an elementary abelian 2-group and $P^{\prime} \leq Z(P)$. Hence $Z(P)=P^{\prime}$.

Corollary 2.1. Let $G$ be a CC-group and $[x]_{\sim}=x^{G}$, for all $x \in G \backslash Z(G)$. Then $G \cong Q_{8}$. 
Proof. By Theorem 2.3, $[x]_{\sim}=x^{G}=x Z(G)$, for all $x \in G \backslash Z(G)$ and $G^{\prime}=Z(G)$. and by Theorem $2.2, G \cong C_{m} \times Q_{8}$ where $m$ is an odd positive integer. Since $G^{\prime}=Z(G)$, so $1 \times Q_{8}^{\prime} \cong C_{m} \times Z\left(Q_{8}\right)$. Therefore $C_{m} \cong 1$. Hence $G \cong Q_{8}$.

Lemma 2.3. A group $G$ is a CA-group and $[x]_{\sim}=x^{G}$, for all $x \in G \backslash Z(G)$ if and only if $|G|=2|Z(G)|^{2}$ and $k(G)=3|Z(G)|-1$.

Proof. Let $G$ be a CA-group and $[x]_{\sim}=x^{G}$, for all $x \in G \backslash Z(G)$. By Theorem 2.3, $[x]_{\sim}=x Z(G)$ for all $x \in G \backslash Z(G)$ and by Lemma 2.1, $C_{G}(x)=[x]_{\sim} \cup Z(G)$, for all $x \in G \backslash Z(G)$. Hence $\left|x^{G}\right|=\frac{|G|}{\left|C_{G}(x)\right|}=\frac{|G|}{|Z(G)|+|[x] \sim|}=\frac{|G|}{2|Z(G)|}$, for all $x \in G \backslash Z(G)$. Since $\left|x^{G}\right|=|x Z(G)|$, for all $x \in G \backslash Z(G)$ we have $|Z(G)|=\frac{|G|}{2|Z(G)|}$ which implies that

$$
|G|=2|Z(G)|^{2} .
$$

Since $[x]_{\sim}=x Z(G)$, for all $x \in G \backslash Z(G)$, by Lemma 2.2 ,

$$
|G|=\frac{2|Z(G)|^{2}}{(3|Z(G)|-k(G))} .
$$

From Equations 2.1 and 2.2 we have $k(G)=3|Z(G)|-1$.

Conversely suppose $|G|=2|Z(G)|^{2}$ and $k(G)=3|Z(G)|-1$. This implies that $|G|=\frac{2|Z(G)|^{2}}{(3|Z(G)|-k(G))}$ and by Lemma $2.2, G$ is a CA-group and $[x]_{\sim}=x Z(G)$ for all $x \in G \backslash Z(G)$. Also by Lemma 2.1, $\left|C_{G}(x)\right|=2|Z(G)|$. This implies that $\left|x^{G}\right|=\frac{|G|}{\left|C_{G}(x)\right|}=\frac{2|Z(G)|^{2}}{2|Z(G)|}=|Z(G)|$. Since $[x]_{\sim}=x Z(G)$, for all $x \in G$, by Lemma 1.3, $|\operatorname{Cent}(G)|=\frac{|G|}{|Z(G)|}$. Hence by Lemma $1.8, \frac{G}{Z(G)}$ is an elementary abelian 2group. Therefore $G^{\prime} \leq Z(G)$ and $x^{G} \subseteq x G^{\prime} \subseteq x Z(G)$, for all $x \in G \backslash Z(G)$. Since $\left|x^{G}\right|=|Z(G)|$, for all $x \in G \backslash Z(G)$, we have $x^{G}=x Z(G)$, for all $x \in G \backslash Z(G)$. Hence we conclude that $[x]_{\sim}=x^{G}=x Z(G)$, for all $x \in G \backslash Z(G)$.

Lemma 2.4. Let $G$ be a CA-group and $[x]_{\sim}=x Z(G)$, for all $x \in G$. Then $[x]_{\sim}=x^{G}$, for all $x \in G \backslash Z(G)$ if and only if $|G|=2|Z(G)|^{2}$.

Proof. Let $G$ be a CA-group and $[x]_{\sim}=x^{G}$, for all $x \in G \backslash Z(G)$. By Lemma 2.3, $|G|=2|Z(G)|^{2}$. Conversely let $|G|=2|Z(G)|^{2}$. Since $G$ is a CA-group and $[x]_{\sim}=x Z(G)$, for all $x \in G$, by Lemma $2.1, C_{G}(x)=Z(G) \cup[x]_{\sim}=Z(G) \cup x Z(G)$, for all $x \in G \backslash Z(G)$. Therefore $\left|C_{G}(x)\right|=2|Z(G)|$, for all $x \in G \backslash Z(G)$. This implies that $\left|x^{G}\right|=\frac{|G|}{\left|C_{G}(x)\right|}=\frac{|G|}{2|Z(G)|}=\frac{2|Z(G)|^{2}}{2|Z(G)|}=|Z(G)|$, for all $x \in G \backslash Z(G)$. Since $[x]_{\sim}=x Z(G)$, for all $x \in G \backslash Z(G)$, by Lemma 1.3 and Lemma $1.8, \frac{G}{Z(G)}$ is an elementary abelian 2-group. Therefore $G^{\prime} \leq Z(G)$. Hence $x^{G} \subseteq x G^{\prime} \subseteq x Z(G)$, for all $x \in G \backslash Z(G)$. Since $\left|x^{G}\right|=|Z(G)|=\mid x Z(G)$, we have $x^{G}=x Z(G)$, for all $x \in G \backslash Z(G)$ and finally $[x]_{\sim}=x^{G}=x Z(G)$ for all $x \in G \backslash Z(G)$.

Example 2.3. Let $G$ be a non-abelian CA-group and assume that $[x]_{\sim}=x^{G}$ for all $x \in G \backslash Z(G)$ and $|G| \leq 100$. Then $G \cong Q_{8}$ or $D_{8}$. 
Lemma 2.5. Let $G$ be a non-abelian group. Then $x^{G}=x Z(G)$, for all $x \in$ $G \backslash Z(G)$ if and only if $G^{\prime}=Z(G)$ and $k(G)=\frac{|G|}{|Z(G)|}+|Z(G)|-1$.

Proof. Let $x^{G}=x Z(G)$, for all $x \in G \backslash Z(G)$. Since $x^{G} \subseteq x G^{\prime}$, so $Z(G) \leq G^{\prime}$. Now we show that $G^{\prime} \leq Z(G)$. Let $1 \neq t \in G^{\prime}$. Then there exist $x, y \in G$ so that $[x, y]=t$. Hence $t=y^{-1} x^{-1} y x=y^{-1} y^{x}$. Since $y^{G}=y Z(G)$, there exists $z \in Z(G)$ such that $y^{x}=y z$. Therefore $t=y^{-1} y^{x}=y^{-1} y z=z$. This implies that $t \in Z(G)$. Thus $G^{\prime} \leq Z(G)$ and we have $G^{\prime}=Z(G)$. Moreover $|G|=|Z(G)|+(k(G)-|Z(G)|)\left|x^{G}\right|$ because $\left|x^{G}\right|=|x Z(G)|$ for all $x \in G \backslash Z(G)$. Hence $\frac{|G|}{|Z(G)|}=k(G)-|Z(G)|+1$ and $k(G)=\frac{|G|}{|Z(G)|}+|Z(G)|-1$.

Conversely, suppose $G^{\prime}=Z(G)$ and $k(G)=\frac{|G|}{|Z(G)|}+|Z(G)|-1$. Then $x^{G} \subseteq$ $x G^{\prime}=x Z(G)$, for all $x \in G \backslash Z(G)$. Hence $\left|x^{G}\right| \leq|x Z(G)|$, for all $x \in G \backslash Z(G)$. Since $k(G)-|Z(G)|=\frac{|G|}{|Z(G)|}-1$ we have $\left|x^{G}\right|=|x Z(G)|$, for all $x \in G \backslash Z(G)$. Therefore $x^{G}=x Z(G)$, for all $x \in G \backslash Z(G)$.

Lemma 2.6. Let $G$ be a non-abelian group and $x^{G}=x Z(G)$, for all $x \in G \backslash Z(G)$. Then $G$ is a p-group where $p$ is a prime.

Proof. Since $\left|x^{G}\right|=|Z(G)|$, for all $x \in G \backslash Z(G)$, so $\Gamma(G)$ is a regular graph. By Lemma $1.2, G \cong A \times P$ where $A$ is an abelian group and $P$ is a $p$-group ( $p$ is a prime). By Lemma $2.5, G^{\prime}=Z(G)$ which implies that $A \cong 1$ and $G$ is a $p$-group.

Theorem 2.4. Let $G$ be a CC-group and $x^{G}=x Z(G)$, for all $x \in G \backslash Z(G)$. Then $G \cong Q_{8}$.

Proof. By Lemma 2.6, $G$ is a $p$-group. So $G$ is a nilpotent group. By Lemma $1.9, G \cong C_{m} \times Q_{2^{n}}$ where $n$ is positive integer and $m$ is an odd positive integer. By Lemma $2.5, G^{\prime}=Z(G)$, so $1 \times Q_{2^{n}}^{\prime} \cong C_{m} \times C_{2}$. Hence $Q_{2^{n}}^{\prime} \cong C_{2}$ and $\left|Q_{2^{n}}^{\prime}\right|=2$. Since $\left|Q_{2^{n}}^{\prime}\right|=2^{n-2}$ we have $n=3$. Hence $G \cong Q_{8}$ and the proof is complete.

\section{Acknowledgements}

The authors were partially supported by Yazd University.

\section{RE F E R E N C E S}

1. A. Abdollahi, S. Akbari and H. R. Maimani: Non commuting graph of group J. Algebra. 28 (2006), 468-492.

2. A. Abdollahi, S. M. Jafarian Amiri and A. M. Hassanabadi: Groups with specific number of centralizers Houston J. Math., 33(1) (2007), 43-57.

3. A. Ashrafi: On finite groups with a given number of centralizers Algebra Colloq. 7(2) (2000), 139-146. 
4. S. M. Belcastro and G. J. Sherman: Counting centralizers in finite groups Math. Mag. 5 (1994), 111-114.

5. M. A. IRANMANESH and M. H. ZAREIAN: On n-centralizer CA-groups submitted.

6. S. M. JAFARIAN Amiri, H. MADAdi and H. Rostami: Finite groups with certain number of centralizers Third Biennial International Group Theory Conference., (2015).

7. S. M. JAFARIAN Amiri and H. Rostami: Finite groups all of whose proper centralizers are cyclic B. Iran. Math. Soc. 43(3) (2017), 755-762.

8. K. PARAttu and A. Wingerter: Tribimaximal mixing from small groups, additonal material Phys. Rev. D, 84(1) 013011. 